\title{
Pathophysiological model of chronic heart failure complicated with renal failure caused by three-quarter nephrectomy and subcutaneous injection of isoprenaline
}

\author{
DING-FENG PENG ${ }^{*}$, SHAO-YONG TANG* ${ }^{*}$, YONG-JUN HU, JIAO CHEN and LI YANG \\ Department of Vasculocardiology, Wuhan Puai Hospital, Wuhan 430033, P.R. China
}

Received October 10, 2012; Accepted November 13, 2012

DOI: $10.3892 /$ etm.2012.865

\begin{abstract}
This study aimed to investigate the pathophysiological changes in a rat chronic heart failure complicated with renal failure model, caused by three-quarters nephrectomy and subcutaneous injection of isoproterenol (ISO). SpragueDawley (SD) rats in the model group received three-quarters nephrectomy after twice undergoing surgical resections and subcutaneous injection of ISO (100 $\mathrm{mg} / \mathrm{kg}$ body weight, injected twice, with a $24 \mathrm{~h}$ interval) after one week, while rats in the control group received sham surgery and injection of normal saline. Survival rate, heart failure and renal failure were compared between the two groups after 4 weeks. Serum creatinine $(\mathrm{Cr})$, blood urea nitrogen (BUN), B-type natriuretic protein (BNP), aldolase (ALD), angiotensin II (Ang II) and C-reactive protein (CRP) were determined by kit assay. Urine protein at $24 \mathrm{~h}$ was determined by the Bradford method and left ventricular systolic pressure (LVSP), left ventricular diastolic pressure (LVDP) and left ventricular end-diastolic pressure (LVEDP), as well as the maximum rates of increased and decreased left ventricular pressure $\left( \pm \mathrm{dP} / \mathrm{dt}_{\max }\right)$ were determined by left ventricular intubation. Heart weight indices were determined and the myocardial pathological conditions were observed by hematoxylin and eosin (HE) staining. There was no death in the control group, while the survival rate of the model group was $73 \%$. Compared with the control group, each index of serum and urine protein in the model group was significantly increased. Additionally, LVSP was decreased, LVDP and LVEDP were increased and heart weight index was increased, with a significant difference. The serum $\mathrm{Cr}$ was positively correlated to BNP levels in the model group. Three-quarters nephrectomy and subcutaneous injection of ISO induces left ventricular heart failure and renal failure
\end{abstract}

Correspondence to: Professor Ding-Feng Peng, Department of Vasculocardiology, Wuhan Puai Hospital, Wuhan 430033, P.R. China E-mail: pdf620630@yahoo.com.cn

*Contributed equally

Key words: isoprenaline, heart failure, renal failure, SpragueDawley rats at the same time, which is characterized in pathophysiology by left ventricular diastolic and systolic function failure, left ventricular myocardial hypertrophy and reconstruction complicated with renal insufficiency.

\section{Introduction}

Cardiorenal syndrome (CRS) is a complex problem. Patients complicated with heart failure and chronic renal insufficiencies are at a greater risk of poor prognosis. The incidences of heart failure and renal failure are gradually increasing, with poorer prognosis. CRS requires greater attention and research to develop therapeutic measures in reference to the fundamental mechanism of this disease. Currently, there are no animal models for studying heart and renal dysfunction. Therefore, it is necessary to establish an effective animal model of heart-kidney interaction in order to increase our understanding of this syndrome.

At present, there are a number of methods for inducing heart $(1,2)$ and renal insufficiency (3), which theoretically could be united to establish a new disease model of heartkidney interaction. However, none of these methods completely represent the pathogenesis and clinical features of human CRS. Therefore, an improved animal model of heart-kidney interaction is required. The pathological changes in an isoproterenol (ISO)-induced congestive heart failure (CHF) model in Sprague-Dawley (SD) rats are characterized by repeated and multiple focal myocardial necrosis, which is similar to ischemic heart disease. There have been reports on varying dosages for creating an ISO-induced CHF model in SD rats (4), in which subcutaneous injection of $>85 \mathrm{mg} / \mathrm{kg}$ ISO is considered a large dose. The large dose has a high success rate of modeling, but with high mortality, while a small dose has a low success rate of modeling, but with greater longevity. The two models are detrimental to drug observation and research. The most common method used to induce renal failure reduces the survival of kidney tissue by different means, resulting in varying extents of removal and severity of renal insufficiency. Unilateral nephrectomy causes slight renal impairment, but without significant urine protein increases and histological changes (5). Renal sub-radical resection leads to more serious renal insufficiency, as well as uremia and complications of chronic kidney disease similar to humans $(6,7)$. It has also 
Table I. Comparison of urine protein, urea and creatinine between the control and model groups.

\begin{tabular}{lccc}
\hline Group & Serum creatinine $(\mu \mathrm{mol} / \mathrm{l})$ & Urea nitrogen $(\mathrm{mmol} / \mathrm{l})$ & Urine protein $(\mathrm{mg} / 24 \mathrm{~h})$ \\
\hline Control & $40.6 \pm 10.8$ & $5.89 \pm 2.26$ & $15.6 \pm 5.36$ \\
Model & $77.5 \pm 8.7^{\mathrm{a}}$ & $10.2 \pm 1.5^{\mathrm{a}}$ & $70.5 \pm 12.7^{\mathrm{a}}$ \\
\hline
\end{tabular}

${ }^{\mathrm{a}} \mathrm{P}<0.01$ vs. the control group. Data are presented as the mean \pm standard deviation.

been reported that renal sub-radical resection causes structural changes to the heart tissues (8-10). This study aimed to explore a new model of CRS and its pathological mechanism.

\section{Materials and methods}

Animals. Thirty SD male rats, weighing $180 \pm 20$ g, provided by the Laboratory Animal Center of Tongji Medical College of Huazhong University of Science and Technology (China) were used in this study. This study was carried out in strict accordance with the recommendations in the Guide for the Care and Use of Laboratory Animals of the National Institutes of Health. The animal use protocol has been reviewed and approved by the Institutional Animal Care and Use Committee (IACUC) of Wuhan Puai Hospital.

Modeling. Thirty SD male rats were randomly divided into a control group $(n=15)$ and model group $(n=15)$. After feeding for one week, the rats in the model group were narcotized with $10 \%$ chloral hydrate $(0.3 \mathrm{ml} / 100 \mathrm{~g}$ intraperitoneal injection). The rats then received resection of the lower pole of the left kidney in the first week and radical resection of the right kidney after one week according to the previously described protocol of twice undergoing surgical resections (11). Rats in the control group underwent surgery twice, although not kidney resection. There was no death in the two groups after conventional feeding for one week. Subcutaneous injections of ISO (100 mg/kg body weight, injected twice) with an interval of $24 \mathrm{~h}$ were administered to the rats in the model group, while rats in the control group received two subcutaneous injections of normal saline (100 mg/ $\mathrm{kg}$ body weight) with an interval of $24 \mathrm{~h}$. All 30 rats received normal water and food for 4 weeks. The general conditions, including diet, hair color, activity, cyanosis and edema were observed and rats were weighed weekly.

Protein determination. After 4 weeks, the rats in the two groups were placed into metabolism cages and urine was collected at $24 \mathrm{~h}$. The Bradford method (Coomassie brilliant blue G-250 staining) was used to detect protein content in the urine and calculate the protein excretion of urine at $24 \mathrm{~h}$. Intraocular venous blood was then collected to prepare serum and the levels of serum creatinine $(\mathrm{Cr})$, blood urea nitrogen (BUN), B-type natriuretic protein (BNP), aldolase (ALD), angiotensin II (Ang II) and C-reactive protein (CRP) was detected.

Hemodynamics. Approximately $24 \mathrm{~h}$ after blood collection, left ventricular intubation was administered via the left common

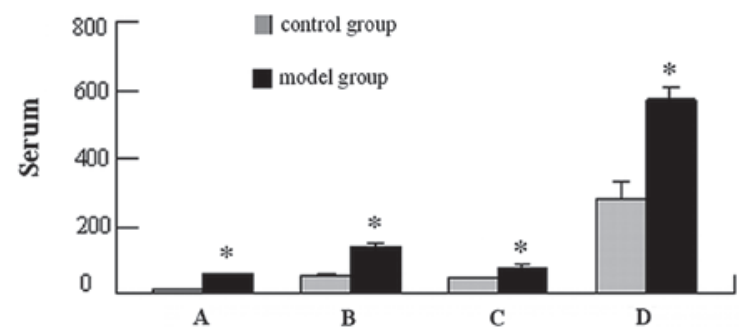

Figure 1. Comparison of BNP, ALD and Ang II between the control and model groups. ${ }^{\mathrm{P}}<0.01$, compared with the control group. (A) Serum BNP (pg/ml); (B) ALD ( $\mu \mathrm{g} / \mathrm{ml})$; (C) Ang II ( $\mu \mathrm{g} / \mathrm{ml})$ and (D) CRP $(\mu \mathrm{g} / \mathrm{ml})$. BNP, B-type natriuretic protein; ALD, aldolase; Ang II, angiotensin II; CRP, C-reactive protein.

carotid artery for left ventricular cardiac function testing. A neck incision was made to expose and bluntly separate the right common carotid artery. The left ventricular catheter was pre-charged with $10 \%$ heparin saline and was intubated against the right common carotid artery into the left ventricle. The other end of the catheter was connected to a pressure transducer (BL-420F biological function experimental system) (Chengdu Technology \& Market Co. Ltd., Chengdu, China) for hemodynamic detection of left ventricular end-diastolic pressure (LVEDP) and left ventricular systolic pressure (LVSP), as well as the maximum rates of increased and decreased left ventricular pressure $\left( \pm \mathrm{dP} / \mathrm{dt}_{\max }\right)$. Changes in cardiac function were calculated.

Hematoxylin and eosin (HE) staining. The left ventricular tissues were fixed in 10X PBS or 4\% neutral formaldehyde. The tissues paraffin-embedded and sliced to produce $4 \mu \mathrm{m}$ sections. The sections underwent HE staining and then images were captured with a Leica microscope (magnification, x100).

Statistical analysis. Data were expressed as the mean \pm standard deviation. Comparison of the mean values between the groups was examined with the $\chi^{2}$ test and variance analysis. Data were analyzed with statistical software SPSS 11.5 (SPSS Inc., Chicago, IL, USA). $\mathrm{P}<0.05$ was considered to indicate a statistically significant difference.

\section{Results}

General information. Compared with the control group, rats in the model exhibited darker hair, reduced feeding, decreased early weight, gradually increased later weight, slow movement, cyanosis in the mouth and nose, asthma, edema in the feet and paws, reduced activity and a poor grab reaction. In the 
Table II. Comparison of left ventricular cardiac function and blood dynamic parameters between the control and model groups.

\begin{tabular}{lccccc}
\hline Group & LVSP $(\mathrm{mmHg})$ & LVDP $(\mathrm{mmHg})$ & LVEDP $(\mathrm{mmHg})$ & $+\mathrm{dP}_{\mathrm{dt}} \mathrm{max}_{(\mathrm{mmHg} / \mathrm{sec})}$ & $-\mathrm{dP} / \mathrm{dt} t_{\max }(\mathrm{mmHg} / \mathrm{sec})$ \\
\hline Control & $136.7 \pm 8.2$ & $0.8 \pm 1.1$ & $2.0 \pm 1.8$ & $8060 \pm 892$ & $-6902 \pm 949$ \\
Model & $110.9 \pm 8.7^{\mathrm{b}}$ & $1.8 \pm 0.6^{\mathrm{a}}$ & $4.6 \pm 1.0^{\mathrm{b}}$ & $5536 \pm 439^{\mathrm{b}}$ & $-4036 \pm 413$ \\
\hline
\end{tabular}

${ }^{\mathrm{a}} \mathrm{P}<0.05$ and ${ }^{\mathrm{b}} \mathrm{P}<0.01$ vs. the control group. Data are presented as the mean \pm standard deviation. LVSP, left ventricular systolic pressure; LVDP, left ventricular diastolic pressure; LVEDP, left ventricular end-diastolic pressure; $\pm \mathrm{dP} / \mathrm{dt}_{\text {max }}$, maximum rates of increased and decreased left ventricular pressure.

Table III. Comparison of weight, left ventricular mass and left ventricular mass index between the control and model groups.

\begin{tabular}{lllr}
\hline Group & LVW $(\mathrm{g})$ & BW $(\mathrm{kg})$ & LVW/BW $(\mathrm{g} / \mathrm{kg})$ \\
\hline Control & $0.40 \pm 0.04$ & $0.29 \pm 0.01$ & $1.38 \pm 0.14$ \\
Model & $0.57 \pm 0.04^{\mathrm{a}}$ & $0.25 \pm 0.01^{\mathrm{a}}$ & $2.29 \pm 0.19^{\mathrm{a}}$ \\
\hline
\end{tabular}

${ }^{a} \mathrm{P}<0.01$ vs. the control group. Data are presented as the mean \pm standard deviation. LVW, left ventricular weight; BW, body weight.

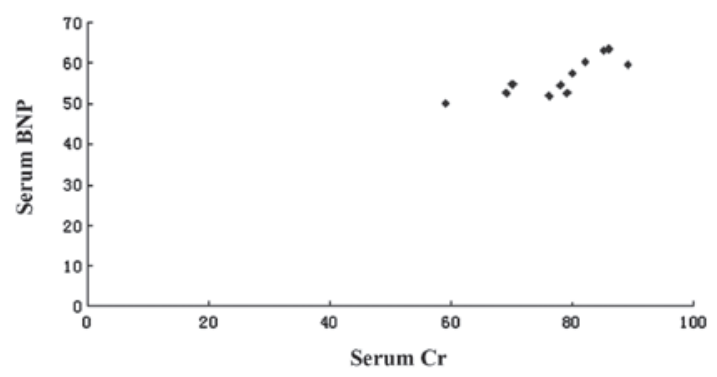

Figure 2. Correlogram of serum BNP and Cr. Serum BNP was positively correlated with serum $\mathrm{Cr}$, with a correlation coefficient $\mathrm{r}=0.81, \mathrm{P}<0.01$. BNP, B-type natriuretic protein; $\mathrm{Cr}$, creatinine.

model group, 3 rats died within 1 week and 4 rats died 4 weeks following the subcutaneous injections of ISO, administered twice, with a survival rate of $73 \%$. No death was noted in the control group.

Cardiac and renal function parameters. Compared with the control group, serum $\mathrm{Cr}$, BUN and urine protein in the model group were increased $(\mathrm{P}<0.01)$, which indicates a successful modeling of renal failure (Table I). All the rats in the model group demonstrated cardiac insufficiency, characterized by significantly decreased LVSP, increased LVDP and LVEDP and decreased $\mathrm{dP} / \mathrm{dt}_{\max }(\mathrm{P}<0.05$; Table II). Compared with $\operatorname{BNP}(13.77 \pm 2.38 \mathrm{pg} / \mathrm{ml}), \operatorname{ALD}(51.57 \pm 9.17 \mu \mathrm{g} / \mathrm{l})$, Ang II $(43.36 \pm 4.63 \mu \mathrm{g} / \mathrm{l})$ and CRP $(282.9 \pm 47.58 \mu \mathrm{g} / \mathrm{l})$ in the control group, the serum BNP $(56.48 \pm 4.67 \mathrm{pg} / \mathrm{ml})$, ALD $(137.69 \pm 16.13 \mu \mathrm{g} / 1)$, Ang II $(81.76 \pm 5.78 \mu \mathrm{g} / 1)$ and CRP $(568.54 \pm 42.15 \mu \mathrm{g} / \mathrm{l})$ were significantly increased in the model group $(\mathrm{P}<0.05$; Fig. 1). Serum $\mathrm{Cr}$ was positively correlated with blood serum BNP levels in the model group (Fig. 2).

Weight index. Compared with the control group, the weights of the rats in the model group were significantly reduced, whereas weights of the left ventricule and ventricular weight indices

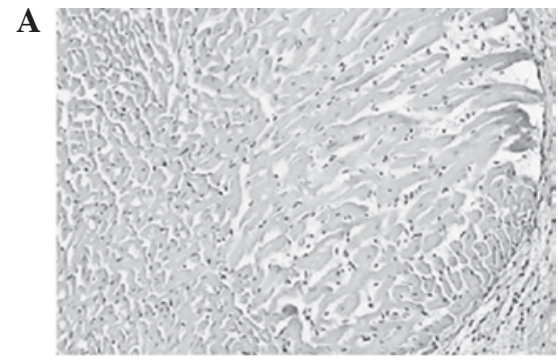

$\mathbf{B}$

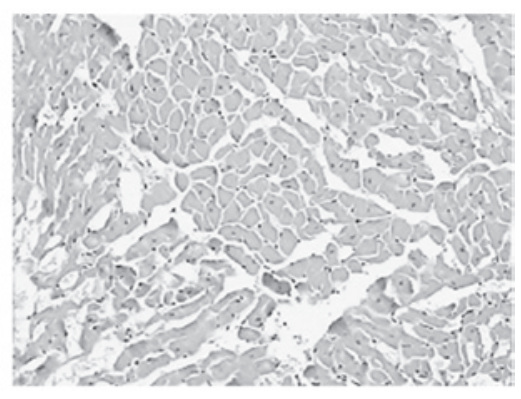

Figure 3. Hematoxylin and eosin (HE) staining of cardiac muscular tissue in the left ventricle. (A) Control group and (B) model group (magnification, $\mathrm{x} 100$ ).

were significantly increased in the model group $(\mathrm{P}<0.01)$. A significant difference was detected between the weight of the left ventricle and the left ventricular weight index $(\mathrm{P}<0.01$; Table III). Cardiac function indices demonstrated that the left ventricle suffered clear hypertrophy and reconstruction and entered the decompensatory stage of heart failure in the model group.

$H E$ staining. Following HE staining, slices were examined and compared with the control group. There was clear cardiomyocyte hypertrophy in the model group, with coarsened myocardial cells in an uneven arrangement and myocardial fibrosis. In the control group, myocardial cells were in even distribution, with fewer and inconspicuous extracellular matrices (Fig. 3). 


\section{Discussion}

The incidences of heart failure and renal failure has gradually increased. Renal insufficiency is an independent predictor of heart failure, while myocardial hypertrophy and heart function failure are serious complications of chronic renal failure, closely associated with mortality (12-14). In this study, a rat model of CRS was established to examine the pathophysiological mechanism in order to identify effective drugs and early intervention therapy.

A successful model of CRS, not only simulates the clinical characteristics of CRS, including changes in the heart, kidney, hemodynamics and neuroendocrinology, but also evaluates the therapeutic effect. This model must include joint damages of kidney and heart, characterized by progressive deterioration of cardio-nephric function. The model must present systolic dysfunction confirmed by echocardiography and haemodynamics, resulting in decreased cardiac output. In addition, to further explore the latest clinical findings, increased enddiastolic pressure and venous congestion are also necessary conditions. At a histological level, the model must present characteristics of hypertrophy and fibrosis, particularly mismatched myocardial/capillary. To successfully represent kidney damage, the model must present characteristics of increased $\mathrm{Cr}$ and excretion of albumin, as well as decreased progressive renal function caused by a decrease in the glomerular filtration rate (GFR)/Cr clearance ratio. This study aimed to explore the physiopathological mechanism of a CRS model induced by three-quarters nephrectomy and subcutaneous injection of ISO.

Compared with previous models of simple heart or renal failure, rats in this study presented earlier renal and heart failure, characterized by significantly increased $\mathrm{Cr}$, urine protein and left ventricular weight index, as well as decreased hemodynamic index, including $\pm \mathrm{dP}^{\mathrm{d} \mathrm{dt}_{\max }}$ and increased serum BNP. HE staining of the myocardium revealed clear hypertrophy of myocardial cells and myocardial fibrosis in the model group, indicating that reconstruction of myocardial hypertrophy had begun. Compared with heart failure caused only by ISO, the amount of subcutaneous injection of ISO was reduced in this model group. In the report of a CRS model by Van Dokkum et al and Windt et al $(5,7)$, there was reduced interactive influence between heart and renal function. Dikow et al (6) hypothesized that the increase in $\mathrm{Cr}$ aggravated the left ventricular remodeling of myocardial infarction. We consider that the differences in experimental design may be associated with the varying degrees of damage to the heart and kidney, as well as different induction times. We analyzed indices of heart and kidney function in this model and identified that serum BNP was positively correlated to $\mathrm{Cr}$ in the model group, with a correlation coefficient of $0.81(\mathrm{P}<0.01)$, which is consistent with the study by Butler et al (15) and the retrospective study by Weinfeld et al (16). The hemodynamic variable associated with worsening renal function was right atrial pressure. Heart and renal function may influence each other in the occurrence and development of diseases. This model simulates the process of heart failure complicated with renal failure.

The possible mechanisms of heart-kidney interactions that have previously been considered include hemodynamic changes, endothelial dysfunction, inflammation, activation of the renin-angiotensin aldosterone system (RAAS) and/or the sympathetic system, any of which may cause cascade reactions of other factors, leading to structural and functional damage to the heart and kidney $(17,18)$. The mechanism that causes and maintains heart-kidney interactions remains unclear. In this study, serum ALD and Ang II were significantly increased in the model group compared with the control group, indicating that activation of the RAAS is important in the occurrence of this model. At the same time, serum CRP was significantly increased in the model group compared with the control group $(\mathrm{P}<0.01)$, indicating that inflammation also plays a promoting role in this model of CRS. We also detected a marked change in urine protein, which is a strong and independent risk factor for cardiovascular disease. When it appears, proteinuria causes an accelerated atherosclerotic process, endothelial dysfunction, increased risk of terminal organ damage, serious cardiovascular events and mortality (19). Rats in this model suffer heart-kidney interactions, then when CRS occurs, the prognosis becomes significantly worse.

Due to the physiological changes of decreased renal function caused by three-quarters nephrectomy, this model had an increased sensitivity to adverse factors in chronic heart failure. Inflammation and activation of the RAAS promotes the occurrence and development of cardiorenal failure. Hemodynamic changes cause an increase in Ang II release, vasoconstriction, contraction of the efferent glomerular arteriole, cardiac remodeling and an increase in aldosterone release, as well as water and sodium retention, which promote myocardial fibrosis (20).

This study demonstrated that three-quarters nephrectomy complicated with subcutaneous injection of ISO induces concurrent heart and renal failure, with a high success rate, providing a simple, reliable and easy animal model for clinical discussion of the interactive mechanism of heart and renal function. However, this study did not investigate the development time of CRS, which is required to examine the illness and possible preventive therapeutic measures. This model is likely to be useful for further studies on the pathogenesis and development of heart-renal interaction at different stages.

\section{References}

1. Balakumar P, Singh AP and Singh M: Rodent models of heart failure. J Pharmacol Toxicol Methods 56: 1-10, 2007.

2. Patten RD and Hall-Porter MR: Small animal models of heart failure: development of novel therapies, past and present. Circ Heart Fail 2: 138-144, 2009.

3. Yang HC, Zuo Y and Fogo AB: Models of chronic kidney disease. Drug Discov Today Dis Models 7: 13-19, 2010.

4. Takeshita D, Shimizu J, Kitagawa Y, et al: Isoproterenolinduced hypertrophied rat hearts: does short-term treatment correspond to long-term treatment? J Physiol Sci 58: 179-188, 2008.

5. Van Dokkum RP, Eijkelkamp WB, Kluppel AC, et al: Myocardial infarction enhances progressive renal damage in an experimental model for cardio-renal interaction. J Am Soc Nephrol 15: 3103-3110, 2004.

6. Dikow R, Schmidt U, Kihm LP, et al: Uremia aggravates left ventricular remodeling after myocardial infarction. Am J Nephrol 32: 13-22, 2010.

7. Windt WA, Henning RH, Kluppel AC, Xu Y, de Zeeuw D and van Dokkum RP: Myocardial infarction does not further impair renal damage in 5/6 nephrectomized rats. Nephrol Dial Transplant 23: 3103-3110, 2008.

8. Amann K, Wiest G, Zimmer G, Gretz N, Ritz E and Mall G: Reduced capillary density in the myocardium of uremic rats - a stereological study. Kidney Int 42: 1079-1085, 1992. 
9. Amann K, Tyralla K, Gross ML, et al: Cardiomyocyte loss in experimental renal failure: prevention by ramipril. Kidney Int 63: 1708-1713, 2003

10. Mall G, Rambausek M, Neumeister A, Kollmar S, Vetterlein F and Ritz E: Myocardial interstitial fibrosis in experimenta uremia - implications for cardiac compliance. Kidney Int 33: 804-811, 1988

11. Tsuruoka S, Nishiki K, Wakaumi M, et al: Chronopharmacology of oxacalcitriol in 5/6 nephrectomized rats. Life Sci 75: 809-822, 2004.

12. Go AS, Chertow GM, Fan D, McCulloch CE and Hsu CY: Chronic kidney disease and the risks of death, cardiovascular events, and hospitalization. N Engl J Med 351: 1296-1305, 2004

13. Sarnak MJ, Levey AS, Schoolwerth AC, et al: Kidney disease as a risk factor for development of cardiovascular disease: a statement from the American Heart Association Councils on Kidney in Cardiovascular Disease, High Blood Pressure Research, Clinical Cardiology, and Epidemiology and Prevention. Hypertension 42: 1050-1065, 2003.

14. Wali RK, Wang GS, Gottlieb SS, et al: Effect of kidney transplantation on left ventricular systolic dysfunction and congestive heart failure in patients with end-stage renal disease. J Am Coll Cardiol 45: 1051-1060, 2005
15. Butler J, Geisberg C, Howser R, et al: Relationship between renal function and left ventricular assist device use. Ann Thorac Surg 81: 1745-1751, 2006.

16. Weinfeld MS, Chertow GM and Stevenson LW: Aggravated renal dysfunction during intensive therapy for advanced chronic heart failure. Am Heart J 138: 285-290, 1999.

17. Bongartz LG, Cramer MJ, Doevendans PA, Joles JA and Braam B: The severe cardiorenal syndrome: 'Guyton revisited'. Eur Heart J 26: 11-17, 2005.

18. Ronco C, Haapio M, House AA, Anavekar N and Bellomo R: Cardiorenal syndrome. J Am Coll Cardiol 52: 1527-1539, 2008.

19. Bongartz LG, Braam B, Verhaar MC, et al: Transient nitric oxide reduction induces permanent cardiac systolic dysfunction and worsens kidney damage in rats with chronic kidney disease. Am J Physiol Regul Integr Comp Physiol 298: R815-R823, 2010.

20. Rajaram V and Joseph J: Role of adenosine antagonism in the cardio-renal syndrome: pathophysiology and therapeutic potential. Curr Heart Fail Rep 4: 153-157, 2007. 